\title{
DESCRIÇÃO E DISCRIMINAÇÃO DE FORMAS DE NERVURAS FOLIARES UTILIZANDO A REPRESENTAÇÃO POLINOMIAL CÚBICA DE HERMITE
}

\author{
Agostinho de Medeiros Brito Júnior \\ Clésio Luis Tozzi ${ }^{1}$ \\ Ana Maria Goulart de Azevedo Tozzi
}

Recebido em 11/9/96. Aceito em 20/11/97

\begin{abstract}
RESUMO- (Descrição e discriminação de formas de nervuras foliares utilizando a representação polinomial cúbica de Hermite). Este artigo descreve um método para caracterização de nervuras utilizando a representação polinomial cúbica de Hermite. Os elementos associados a esta representação são usados como descritores das nervuras e o potencial discriminatório é analisado com base na identificação de duas espécies de leguminosas (Lonchocarpus muehlbergianus Hassl. e L. subglaucescens Mart. ex Benth.). Os vetores de geometria de Hermite exercem influência sobre toda a extensão da curva modelada, permitindo a descrição global da nervura por um descritor de baixa dimensionalidade. Os resultados obtidos mostram que o método foi eficaz na separação das espécies analisadas e apresenta-se como uma opção adicional aos métodos para caracterização de nervuras usualmente utilizados em taxonomia vegetal.
\end{abstract}

Palavras-chave: Lonchocarpus, venação foliar, descritores de forma, classificação

\begin{abstract}
Leaf vein shape description and discrimination using Hermite cubic polynomial representation). This paper describes a method for leaf vein shape characterization using Hermite polynomial cubic representation. The elements associated with this representation are used as secondary vein descriptors and their discriminatory potential are analyzed based on the identification of two legume species (Lonchocarpus muehlbergiamus Hassl. and L. subglaucescens Mart. ex Benth.). The elements of Hermite geometry influence a curve along all its extension allowing a global description of the secondary vein course by a descriptor of low dimensionality. The obtained results shown the analyzed species can be discriminated by this method and it can be used in addition to commonly considered elements in the taxonomic process.
\end{abstract}

Key words: Lonchocarpus, leaf venation, shape descriptors, classification

\footnotetext{
' Universidade Estadual de Campinas, Faculdade de Engenharia Elétrica e de Computação, Departamento de Computação e Automação Industrial. C.P. 6101, CEP 13083-970, Campinas, SP

2 Universidade Estadual de Campinas, Instituto de Biologia, Departamento de Botânica. C.P. 6109, CEP13083-970, Campinas, SP
} 


\section{Introdução}

O processo de identificação constitui-se em um dos objetivos básicos da taxonomia vegetal e uma das ferramentas mais utilizadas neste processo é a chave diagnóstica. Monografias e Floras incorporam chaves através das quais o leitor pode identificar uma planta desconhecida com uma incluída no trabalho em questão.

As chaves não fornecem uma descrição das plantas, mas mostram os caracteres diagnósticos essenciais, por meio dos quais os táxons podem ser identificados. A escolha desses caracteres é de fundamental importância na elaboração da chave e a presença desses é essencial para a identificação. Assim, o uso de características vegetativas como elementos diagnósticos para espécimes vegetais têm sido considerados cada vez mais relevantes no processo de identificação, em especial daquelas relacionadas à forma- das partes constituintes do vegetal. Embora a forma há muito tempo seja aceita como elemento importante no estudo taxonômico, a sua aplicação não tem sido explorada em todo seu potencial, devido à dificuldade inerente de sua descrição e comparação (Niklas 1994).

A folha é um dos órgãos vegetais que, apresentando ampla faixa de variação morfológica, se enquadra nas condições anteriormente descritas. As características relacionadas à forma da lâmina, principalmente à forma do ápice e da base, têm sido consideradas através da utilização de termos padronizados (Dickinson et al. 1987). No entanto, alguns caracteres morfológicos das folhas, que poderiam contribuir para a melhor utilização de seu potencial taxonômico, têm sido menos considerados devido à limitação ou subjetividade associadas à sua descrição.

Entre estes caracteres morfológicos encontram-se as formas das nervuras foliares. A plasticidade e a variabilidade da venação da folha dentro de um mesmo indivíduo têm sido estudadas ontogeneticamente e, embora as nervuras e os padrões de venação possam ser influenciados por fatores ambientais, o padrão básico de venação não varia muito dentro de uma única espécie, o que o torna valioso para a sistemática (Köhler 1993, apud Roth et al. 1995). Tal situação pode ser verificada de forma intuitiva para Lonchocarpus muehlbergianus Hassl. e L. subglaucescens Mart. ex Benth., onde, aparentemente, o curso das nervuras secundárias poderia ser um provável carácter separador das espécies. Todavia, a formalização desta característica como elemento de diagnóstico apresenta dificuldades para a sua descrição e quantificação.

Dentre os poucos trabalhos que tratam da descrição e classificação da venação de folhas destacam-se os elaborados por Hickey $(1973,1979)$, Melville $(1969,1976)$ e Rizzini (1977). Nestes trabalhos, a descrição da venação se limita a uma caracterização qualitativa do curso das nervuras, da localização do ponto de inserção e da terminação, do comprimento relativo e da espessura, além das medidas dos ângulos de inserção. Estas medidas, em geral, apresentam resolução limitada, visto que são feitas dentro de faixas muito largas, resultando numa quantificação pouco precisa e, conseqüentemente, de menor importância no processo taxonômico (Hickey 1979). Além disso, tais medidas são de natureza local, não refletindo o curso das nervuras como um todo.

Nas últimas décadas, com a disponibilidade de meios eletrônicos de aquisição de dados, trabalhos na área de visão computacional que tratam do reconhecimento, 
classificação ou identificação, fundamentados em descrições quantitativas e globais da forma, têm sido apresentados na literatura (Bookstein et al. 1982; West \& Noble 1984; Ferson et al. 1985; Lin \& Hwang 1987; Dickinson et al. 1987; McLellan 1993; Kores et al. 1993; Heijden \& Berg 1997).

Para a descrição da forma são apresentados e discutidos pelos diversos autores o uso de diferentes descritores. Dentre estes descritores, o que tem apresentado maior aplicação, tanto em biologia como em outras áreas de conhecimento, é o descritor de Fourier, que é aplicado a formas ou curvas fechadas, embora seu uso possa ser estendido para curvas abertas. O custo computacional na determinação dos coeficientes é relativamente alto e, para efeitos de discriminação, grande número de coeficientes pode eventualmente ser necessário. Assim, tendo como objetivo a busca de descritores alternativos de menor custo computacional e de efetiva capacidade discriminatória, propõe-se neste trabalho o uso dos elementos da representação polinomial cúbica de Hermite (Foley et al. 1990) como descritores para caracterização do curso das nervuras secundárias foliares.

\section{Material e métodos}

Para avaliação da representação proposta foi considerada sua aplicação na discriminação, com base na descrição das nervuras secundárias, de duas espécies afins e simpátricas de leguminosas (Lonchocarpus muehlbergianus e $L$. subglaucescens). Estas espécies apresentam grande semelhança vegetativa e nítida sobreposição nos estados de caracteres tradicionalmente analisados, com folhas imparipinadas, geralmente 9-folioladas, folíolos elípticos ou oblongos (Fig. 1), os basais ovais e o terminal oboval, de 4 a $12 \mathrm{~cm}$ de comprimento e 1,5 a $6 \mathrm{~cm}$ de largura.

Foram analisadas 76 amostras de folíolos (recolhidos aleatoriamente de indivíduos diferentes e em condições ambientais diversas), sendo 38 de $L$. subglaucescens e 38 de L. muehlbergianus. Em todos os casos, a aquisição dos dados foi realizada no terceiro par de folíolos contados a partir da base da folha.

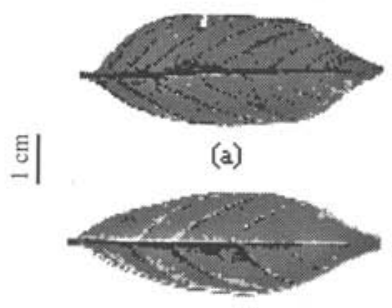

(c)

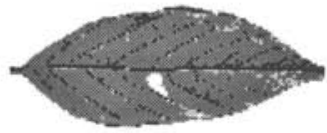

(b)

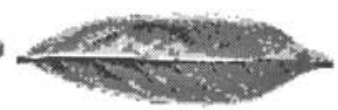

(d)

Figura 1. Folíolos digitalizados de Lonchocarpus muehlbergianus (a, b) e L. subglaucescens (c, d). 
A partir de imagens digitalizadas de folíolos destas espécies, foi realizado um processo formal de análise do curso das nervuras com base no descritor proposto. Embora no presente trabalho a metodologia tenha sido aplicada aos folíolos, ela pode ser aplicada, indistintamente, a folhas e folíolos.

Técnicas de aproximação de curvas - As nervuras podem ser vistas como curvas bidimensionais e, para que sua modelagem possa ser realizada, diversas abordagens são possiveis, tais como: equações polinomiais explícitas, curvas de Bézier, splines cúbicas, B-splines, curvas parabólicas, todas descritas em Rogers \& Adams (1990), e também abordagens onde a modelagem de curvas é feita através de polinômios ortogonais (Zhou et al. 1985).

A representação de Hermite é uma representação alternativa de splines cúbicas, que são curvas paramétricas expressas sob a forma $Q(t)=\left[\begin{array}{ll}x(t) & y(t)\end{array}\right]$, onde $x(t)$ e $y(t)$ são funções cúbicas paramétricas em $t$ e cada valor $t=t_{i}$ define um ponto $\left(x_{i}, y_{i}\right)$ sobre a curva.

A opção pela representação da forma da nervura por funções paramétricas de terceiro grau, a de Hermite em particular, se deu pela flexibilidade no controle da forma da curva. Funções de grau inferior não permitem ajuste adequado da forma, enquanto funções de grau superior, além de proporcionarem ondulações indesejadas na forma da curva, requerem custo computacional maior (Foley et al. 1990).

Com o objetivo de facilitar a compreensão da solução proposta, é apresentada a seguir breve revisão de conceitos relacionados à representação polinomial de curvas.

Representação paramétrica - As equações polinomiais cúbicas que definem uma curva genérica $Q(t)$ são do tipo:

$$
\left\{\begin{array}{l}
\mathrm{x}(\mathrm{t})=a_{x} t^{3}+b_{x} t^{2}+c_{x} t+d_{x} \\
\mathrm{y}(\mathrm{t})=a_{y} t^{3}+b_{y} t^{2}+c_{y} t+d_{y}
\end{array}\right.
$$

Uma representação mais compacta das equações 1 é obtida por intermédio de uma equação matricial da forma

$$
Q(t)=T \cdot C
$$

onde:

$$
T=\left[\begin{array}{llll}
t^{3} & t^{2} & t & 1
\end{array}\right]
$$

$\mathrm{e}$

$$
C=\left[\begin{array}{ll}
a_{x} & a_{y} \\
b_{x} & b_{y} \\
c_{x} & c_{y} \\
d_{x} & d_{y}
\end{array}\right]=\left[\begin{array}{ll}
C_{x} & C_{y}
\end{array}\right]
$$


Representação na forma de Hermite - Para uma curva representada na forma de Hermite, o segmento polinomial é determinado pelos valores dos seus pontos finais $P_{l}$ e $P_{2}$ e dos vetores tangentes à curva nestes pontos, $R_{l}$ e $R_{2}$, onde $t$ é normalizado, assumindo valores no intervalo $[0,1]$. Estes vetores são denominados vetores de geometria da curva e estão representados na Fig. 2.

Os vetores de geometria podem ser expressos em função do vetor de posição

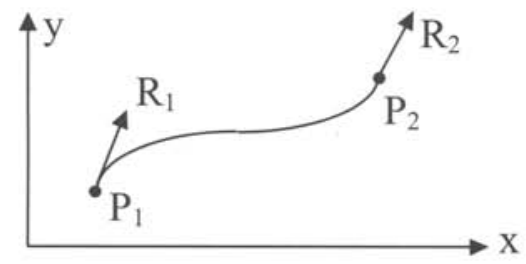

Figura 2. Curva na representação de Hermite com os vetores de geometria $P_{1}, P_{2}, R_{1} e R_{2}$.

$Q(t)$, como descrevem as equações 5 .

$$
\begin{array}{r}
P_{1}=\left[\begin{array}{ll}
P_{1 x} & P_{1 y}
\end{array}\right]=Q(0) \\
P_{2}=\left[\begin{array}{ll}
P_{2 x} & P_{2 y}
\end{array}\right]=Q(1) \\
R_{1}=\left[\begin{array}{ll}
R_{1 x} & R_{1 y}
\end{array}\right]=Q^{\prime}(0) \\
R_{2}=\left[\begin{array}{ll}
R_{2 x} & R_{2 y}
\end{array}\right]=Q^{\prime}(1)
\end{array}
$$

onde:

$$
Q^{\prime}(t)=d Q(t) / d t=\left[\begin{array}{llll}
3 t^{2} & 2 t & 1 & 0
\end{array}\right] \cdot C
$$

Definindo a matriz de geometria

$$
G_{H}=\left[\begin{array}{ll}
P_{l x} & P_{l y} \\
P_{2 x} & P_{2 y} \\
R_{l x} & R_{l y} \\
R_{2 x} & R_{2 y}
\end{array}\right]
$$

é possível mostrar que o vetor de posição $Q(t)$ pode ser escrito na forma

$$
Q(t)=[x(t) \quad y(t)]=T \cdot C=B_{H} \cdot G_{H}
$$


onde os elementos da matriz

$$
B_{H}=\left[\begin{array}{l}
2 t^{3}-3 t^{2}+1 \\
-2 t^{3}+3 t^{2} \\
t^{3}-2 t^{2}+t \\
t^{3}-t^{2}
\end{array}\right]^{T}
$$

são denominados funções de blending de Hermite (Fig. 3) e ponderam a influência de cada elemento da matriz $G_{H}$ ao longo da curva $Q(t)$.

É importante salientar que todos os elementos da representação de Hermite são importantes na representação da curva. Caso apenas um deles seja alterado, toda a forma da curva será influenciada por esta alteração. Esta afirmação pode ser verificada pela observação das Fig. 4a a 4d. Elas mostram situações onde um dos elementos é alterado, enquanto os demais permanecem constantes. Nas Fig. 4a e 4b, apenas os vetores de geometria $\mathrm{P}_{1}$ e $\mathrm{P}_{2}$ são alterados, respectivamente, enquanto que nas Fig. $4 c$ e $4 d$ as alterações ocorrem em $R_{1}$ e $R_{2}$, respectivamente. Nestes últimos dois casos, as associações entre as curvas e os correspondentes vetores de geometria são feitas pelo tipo de tracejado no desenho. Em todas as situações, pode ser constatado que a forma da curva muda mesmo que apenas um único elemento da representação seja alterado, o que reforça a idéia de que todos os elementos dos vetores de geometria devem ser levados em consideração na análise da curva.

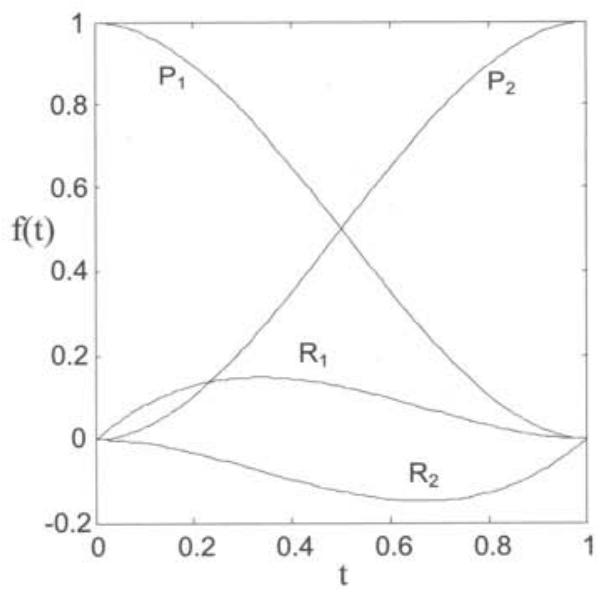

Figura 3. Funções de blending de Hermite associadas aos elementos do vetor de geometria $G_{H}$. 


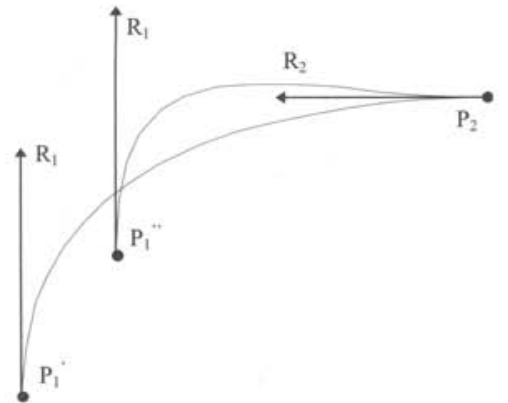

(a)

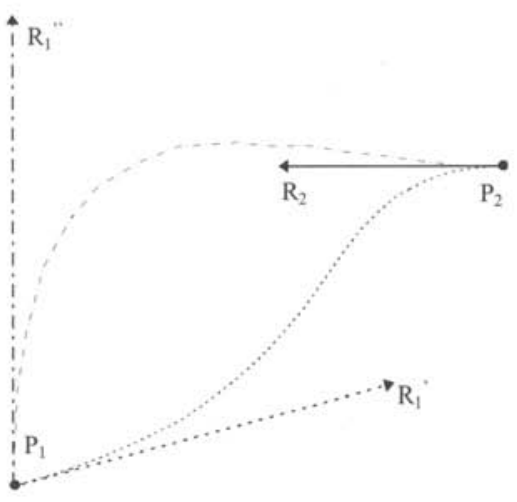

(d)

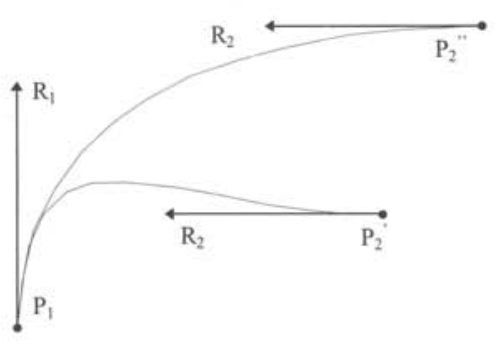

(b)

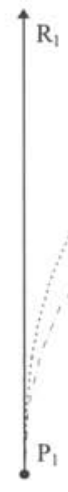

(e)

Figura 4. Exemplos de curvas na representação de Hermite para diferentes combinações de vetores de geometria. Em cada exemplo, apenas um dos vetores é alterado enquanto que os outros permanecem iguais. (a) alteração em $\mathrm{P}_{1}$; (b) alteração em $\mathrm{P}_{2}$; (c) alteração em $\mathrm{R}_{1}$; (d) alteração em $\mathrm{R}_{2}$.

Ajuste polinomial pelo método dos mínimos quadrados - Dada uma curva real, o que se procura neste contexto é a determinação dos parâmetros da matriz de geometria que melhor representa esta curva. Duas formas para determinação destes parâmetros podem ser consideradas. A primeira delas consiste no ajuste iterativo dos módulos e direções dos vetores de geometria, até a obtenção da aproximação desejada para a curva real. Esta abordagem não é recomendada, pois exige um trabalho exaustivo para o ajuste e depende da experiência de quem o realiza. A segunda forma é feita pela amostragem de pontos sobre a curva e os vetores de geometria obtidos através da minimização do erro entre a curva real e a curva modelada. Esta última abordagem, por ser a mais apropriada, foi implementada neste trabalho e os aspectos relacionados a sua implementação são apresentados na seqüência. 
O ajuste da curva a ser modelada é feito através do método dos mínimos quadrados (Press et al. 1992), sendo os seus parâmetros ideais obtidos através da minimização de uma função de erro entre a curva modelada e a curva real.

Ajuste polinomial para uma função explícita $-\mathrm{O}$ ajuste polinomial consiste em encontrar para um polinômio de grau $p$, o conjunto de coeficientes $b_{j}$ que minimiza a função de erro para um conjunto de $\mathrm{N}$ pontos com coordenadas $\left(x_{i}+y_{i}\right), i=1, \ldots, N$, amostrados sobre a curva. $\mathrm{O}$ valor ajustado मf r relativo a $x_{i}$ é dado pela equação 10 .

$$
\text { म }=b_{0}+b_{1} x_{i}+b_{2} x_{i}^{2}+\mathrm{K}+b_{p} x_{i}^{P}
$$

Definindo o erro

$$
e_{i}=y_{i}-\text { A }
$$

e a função de erro $\xi=\mathrm{F}\left(\mathrm{b}_{1}, \mathrm{~b}_{2}, \mathrm{~K}, \mathrm{~b}_{\mathrm{P}}\right)$ como o somatório dos erros quadráticos $e_{i}$, ou seja,

$$
\xi=\sum_{i=1}^{N} e_{i}^{2}=e_{1} e_{1}+e_{2} e_{2}+\Lambda+e_{N} e_{N}
$$

o valor mínimo para a expressão do erro quadrático é obtido pela determinação das derivadas parciais da equação 12 em relação a cada elemento $b_{i}$ e igualando os resultados a zero. Na forma matricial, esta condição é expressa pela equação

$$
\left[\begin{array}{ccccc}
s_{0} & s_{1} & s_{2} & \Lambda & s_{P} \\
s_{1} & s_{2} & s_{3} & \Lambda & s_{P+1} \\
s_{2} & s_{3} & s_{4} & \Lambda & s_{P+2} \\
\mathrm{M} & \mathrm{M} & \mathrm{M} & \mathrm{O} & \mathrm{M} \\
s_{P} & s_{P+1} & s_{P+2} & \Lambda & s_{2 P}
\end{array}\right] \cdot\left[\begin{array}{c}
b_{0} \\
b_{1} \\
b_{2} \\
\mathrm{M} \\
b_{P}
\end{array}\right]=\left[\begin{array}{c}
k_{o} \\
k_{1} \\
k_{2} \\
\mathrm{M} \\
k_{P}
\end{array}\right]
$$

onde:

$$
s_{j}=\sum_{i=1}^{N} x_{i}^{j} \text { e } k_{j}=\sum_{i=1}^{N} y x_{i}^{j}
$$

Ajuste de curvas para um modelo paramétrico - Para uma representação polinomial paramétrica, a abordagem apresentada no item anterior deve ser modificada de modo que as equações paramétricas $x(t)$ e $y(t)$ sejam dependentes e ajustem adequadamente a curva no plano $x y$. Conseqüentemente, os ajustes das duas curvas $[t, x(t)]$ e $[t, y(t)]$, devem ser efetuados iterativamente.

Além disso, para o modelo polinomial de Hermite, o método dos mínimos quadrados foi modificado de modo a introduzir restrições, garantindo que os pontos inicial e final da curva modelada coincidissem com os pontos inicial e final da curva real, respectivamente. 
Para o ajuste das curvas paramétricas $x(t)$ e $y(t)$ é necessário que o parâmetro $t$ seja estabelecido. Os valores ideais de $t_{i}$ associados a cada ponto amostrado $\left(x_{i}, y_{i}\right)$ são determinados pelo algoritmo de otimização proposto por Plass \& Stone (1983), que minimiza o erro entre a curva modelada e os pontos amostrados.

O algoritmo é iterativo e utiliza inicialmente uma distribuição uniforme para os valores do parâmetro $t_{i}$ e ajusta as curvas $x(t)$ e $y(t)$ pelo método dos mínimos quadrados. Em cada iteração seguinte são calculados, pelo método de Newton-Raphson (Press et al. 1992), os valores de $t_{i, n+1}$ (onde $t_{i, n}$ é o valor do parâmetro $t_{i}$ na iteração n) que minimizam o quadrado da distância,

$$
d_{i, n+1}^{2}=\left(x_{i}-x\left(t_{i, n+1}\right)\right)^{2}+\left(y_{i}-y\left(t_{i, n+1}\right)\right)^{2}
$$

de cada ponto amostrado à curva modelada.

Com estes novos valores de $t_{i}$, o método dos mínimos quadrados é utilizado para o ajuste das novas curvas $[\mathrm{t}, \mathrm{x}(\mathrm{t})] \mathrm{e}[\mathrm{t}, \mathrm{y}(\mathrm{t})]$, sendo este processo de ajuste repetido até que um critério de convergência seja satisfeito. O critério aqui adotado foi o de estabilização do somatório de todos os erros $d_{i}^{2}$, significando que a curva chegou ao seu melhor ajuste.

Aquisição dos dados e descrição das nervuras - A aquisição dos dados e a determinação dos elementos da matriz de geometria que caracterizam as curvas correspondentes às nervuras foliolares secundárias analisadas foram realizadas por intermédio de software desenvolvido pelos autores, sendo procedidas conforme as fases a seguir. A Fig. 5 mostra uma representação de um folíolo de Lonchocarpus, destacando os componentes de interesse para a aplicação.

Fase 1 - Aquisição de dados - Nesta fase é colocada sobre a imagem do folíolo uma reta que aproxima sua nervura principal. Esta reta serve de referência para determinação da transformação de rotação que alinha o eixo principal do folíolo com o eixo horizontal do dispositivo de exibição. Os pontos são então marcados sobre as nervuras, tal como observado na Fig. 6.

Inicialmente foi considerada a amostragem sobre toda a extensão da nervura secundária. Todavia, em função de análises preliminares dos resultados, foi verificado que a parte final da curva apresenta baixo potencial discriminatório. Desta forma, apenas a parte inicial da curva, correspondente a um terço da projeção da curva no eixo horizontal, foi considerada. Isto permitiu que as nervuras fossem melhor ajustadas por polinômios do terceiro grau e a obtenção de maior potencial discriminatório para os elementos da representação.

Fase 2 - Modelagem das curvas e obtenção de descritores-Para o ajuste das curvas foram estabelecidos sistemas referenciais para cada nervura (Fig. 5). De modo a manter consistência entre os sistemas referenciais de todas as nervuras secundárias e possibilitar a análise dos dados modelados, os eixos horizontais dos sistemas referenciais estabelecidos para cada nervura são coincidentes com a nervura principal do folíolo, tendo o sentido base $\rightarrow$ ápice, e os eixos verticais de cada 


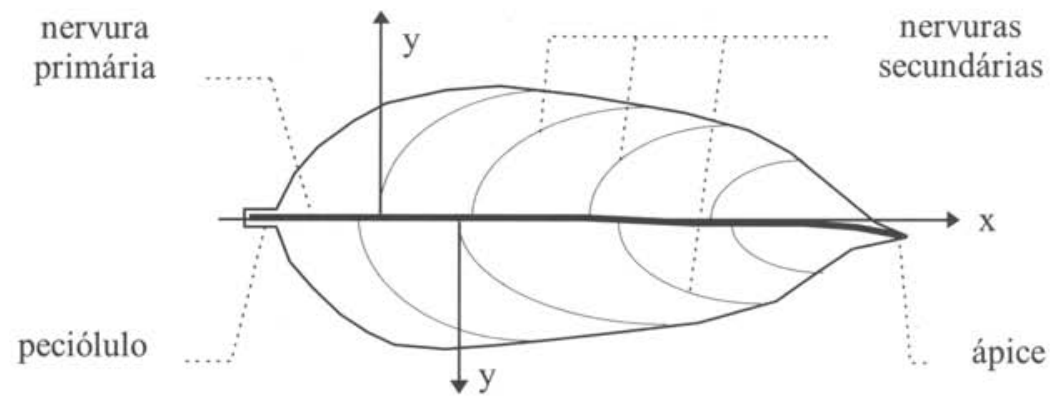

Figura 5. Estrutura de um foliolo e os eixos referenciais considerados.

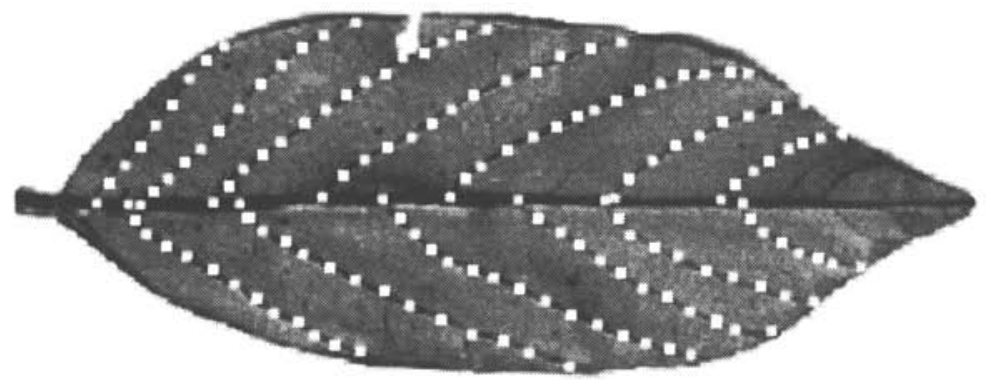

Figura 6. Imagem digitalizada de um folíolo com a marcação dos pontos amostrados.

nervura o sentido nervura principal $\rightarrow$ borda lateral correspondente. A origem dos eixos, para cada nervura modelada, é colocada no ponto de inserção da nervura secundária analisada.

Para obtenção de um descritor invariante com a escala (i.e., independente do tamanho do folíolo), foi adotada a normalização da curva através de escalonamento global nas coordenadas dos pontos amostrados, de modo a colocá-los numa faixa $x \in[0,1]$ e $y \in[0,1]$. Portanto, quando a projeção horizontal da nervura for maior que sua projeção vertical, o valor máximo de $x$ será 1 enquanto o valor máximo de y estará situado entre 0 e 1 , e vice-versa. 
A caracterização do folíolo é feita por uma única nervura, que é escolhida com base no vetor de geometria $\mathrm{R}_{1}$. Uma transformação da forma

$$
\theta_{\mathrm{k}}=\left\langle\mathrm{R}_{1}\right\rangle=\tan ^{-1}\left(\mathrm{R}_{1 y} / \mathrm{R}_{1 x}\right)
$$

é realizada e o valor de $\theta_{\mathrm{k}}$ calculado para todas as curvas representativas das nervuras do folíolo. A nervura associada ao valor mediano para $\theta_{\mathrm{k}}$ é considerada a nervura representativa do folíolo.

Calculados os vetores de geometria com base na nervura representativa do folíolo foi analisado o potencial de discriminação para a representação proposta. O critério separador de classes utilizado foi o discriminante linear de Fisher associado à teoria de Bayes (Schalkoff 1992). O método de Fisher determina uma reta no espaço ndimensional, cuja orientação assegura que os conjuntos dos dados projetados ortogonalmente na mesma tenham a máxima separação possível. Tal orientação é calculada pela maximização de uma função que relaciona as médias e variâncias projetadas das amostras, como expresso na equação 16 .

$$
J=\frac{\left(\bar{m}_{Y 1}-\bar{m}_{Y 2}\right)^{2}}{s_{Y 1}^{2}+s_{Y 2}^{2}}
$$

Foi assumido distribuição normal $N\left(\mu_{l}, \sigma_{l}\right)$ para cada conjunto de dados projetados na reta correspondente a cada par de classes consideradas. Utilizando intervalos de confiança (Box \& Hunter 1978) de 95\% para as médias e para as variâncias foi calculado, pelo método de Bayes, o ponto de separação entre classes na reta de projeção.

\section{Resultados e discussão}

O potencial de discriminação para o conjunto de dados adquiridos das 78 amostras foi analisado considerando as seguintes combinações dos vetores de geometria:

1. os vetores de geometria $\mathrm{R}_{1}$ e $\mathrm{R}_{2}$ e $\mathrm{P}_{2}$;

2. os vetores de geometria $R_{1}$ e $R_{2}$;

3. os vetores de geometria $R_{1}$ e $P_{2}$;

4. somente o vetor de geometria $\mathrm{R}_{1}$;

5. somente o vetor de geometria $\mathrm{R}_{2}$; e

6. somente o vetor de geometria $\mathrm{P}_{2}$.

$\mathrm{O}$ vetor de geometria $\mathrm{P}_{1}$ não foi considerado na análise visto que, sendo este coincidente com a origem dos sistemas referenciais, resulta em um vetor nulo para todas as amostras, não contribuindo para a separação das classes.

Nas Fig. 7 a 9 são apresentados os gráficos obtidos para os dados projetados considerando as proposições 1 a 3 e nas Fig. 10 a 12 são mostradas as componentes dos vetores de geometria $\mathrm{R}_{1}, \mathrm{R}_{2}$ e $\mathrm{P}_{2}$, respectivamente. Na Fig.10, que apresenta as componentes vetoriais de $\mathrm{R}_{1}$, é mostrada também a reta discriminante entre as classes. $\mathrm{O}$ coeficiente linear da reta discriminante no espaço bidimensional foi determinado a partir do ponto de separação na reta de projeção. 
Observa-se das Fig. 7 a 12 que as quatro primeiras combinações dos vetores de geometria (proposições 1 a 4) possuem capacidade discriminatória, ou seja, é possível a identificação das espécies pelos elementos descritores considerados. Todavia, é necessária a determinação do erro de classificação inerente a cada uma das proposições, no sentido de determinar o desempenho e a influência de cada elemento descritor no processo discriminatório.

Esta análise, para as proposições 1 a 4, foi realizada com base na probabilidade de classificação errada, isto é, na probabilidade de um folíolo pertencente à espécie $\mathrm{A}$ ser determinado como sendo pertencente à espécie B e vice-versa. Tais probabilidades foram calculadas com base nas estimativas da média e variância do conjunto dos

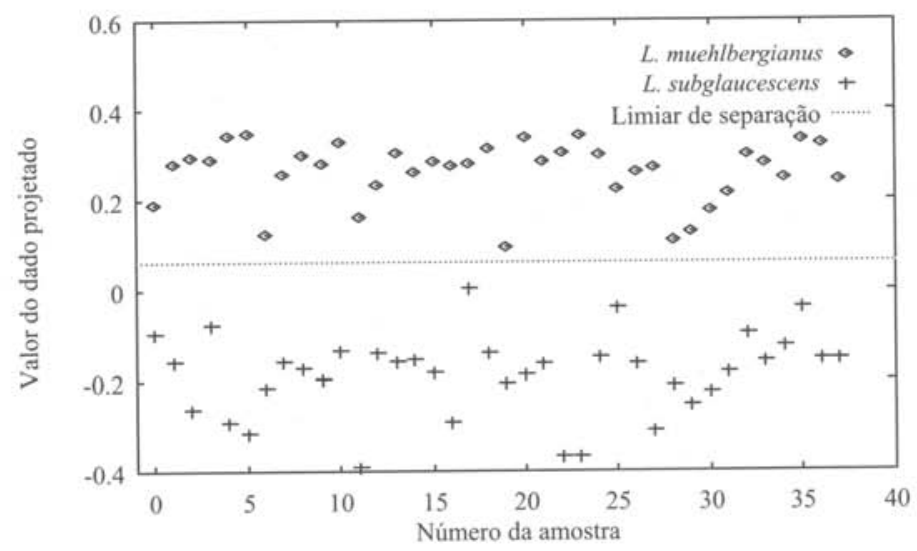

Figura 7. Valores das projeções sobre a reta determinada pelo método de Fisher/Bayes para os vetores de geometria $\mathrm{R}_{1}, \mathrm{R}_{2}, \mathrm{P}_{2}$.

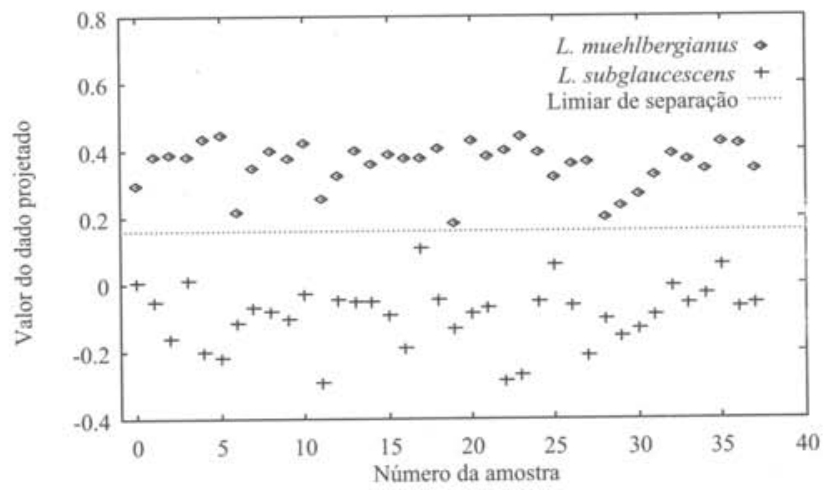

Figura 8. Valores das projeções sobre a reta determinada pelo método de Fisher/Bayes para os vetores de geometria $\mathrm{R}_{1}, \mathrm{R}_{2}$. 


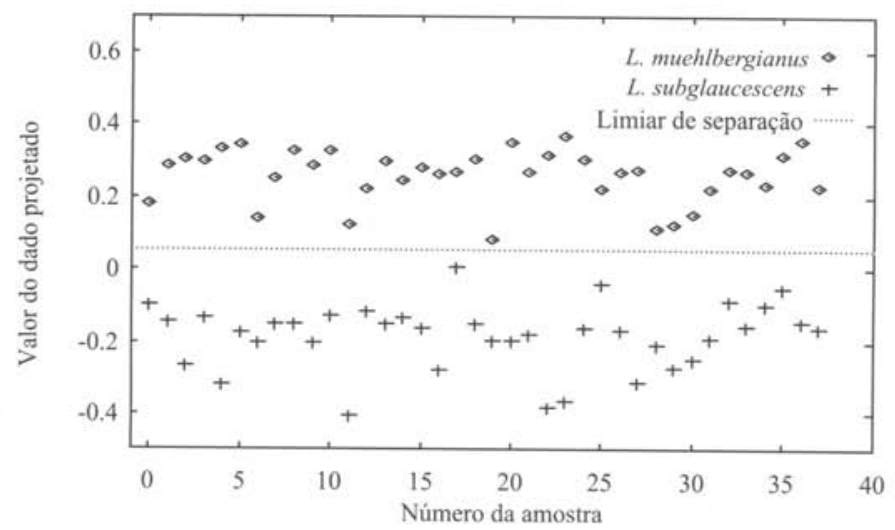

Figura 9. Valores das projeções sobre a reta determinada pelo método de Fisher/Bayes para os vetores de geometria $\mathrm{R}_{1} \mathrm{e} \mathrm{P}_{2}$.

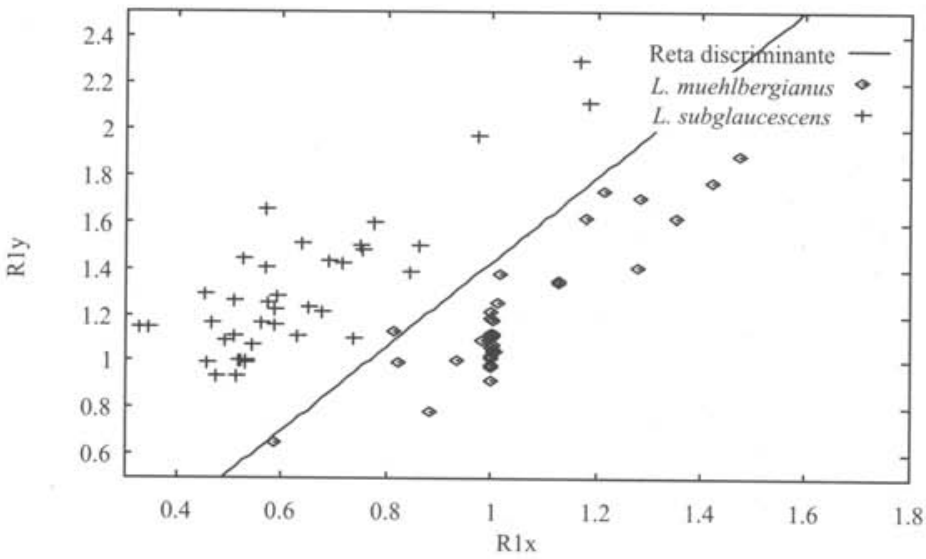

Figura 10. Componentes do vetor de geometria $\mathrm{R}_{\mathrm{I}}$.

dados projetados, assumindo distribuição normal $N\left(\mu_{i}, \sigma_{i}\right)$, e do ponto de separação entre classes na reta de projeção, determinado utilizando intervalos de confiança de $95 \%$ para as médias e para as variâncias. Os resultados obtidos, correspondentes às máximas probabilidades de erro de identificação para as espécies, são apresentados na Tab. 1.

A análise dos dados da Tab.1 mostra que as combinações dos elementos da geometria considerados nas proposições 1 a 4 são apropriados para discriminação das espécies Lonchocarpus muehlbergianus e $L$. subglaucescens com erros inferiores a $5 \%$.

Observa-se que para os casos 1 a 3 foi feita uma redução da dimensionalidade utilizando o discriminante linear de Fisher, enquanto no caso 4 a separação entre 


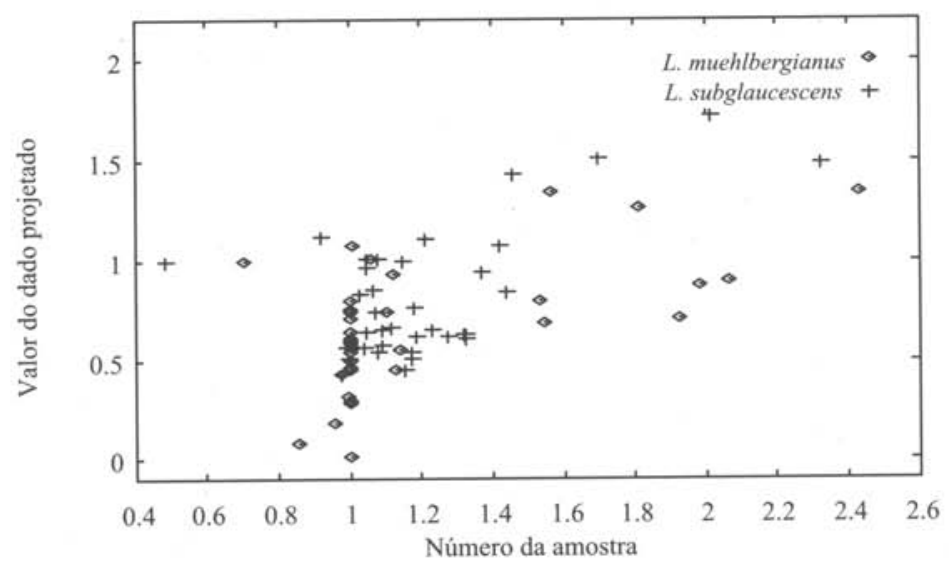

Figura 11. Componentes do vetor de geometria $\mathrm{R}_{2}$.

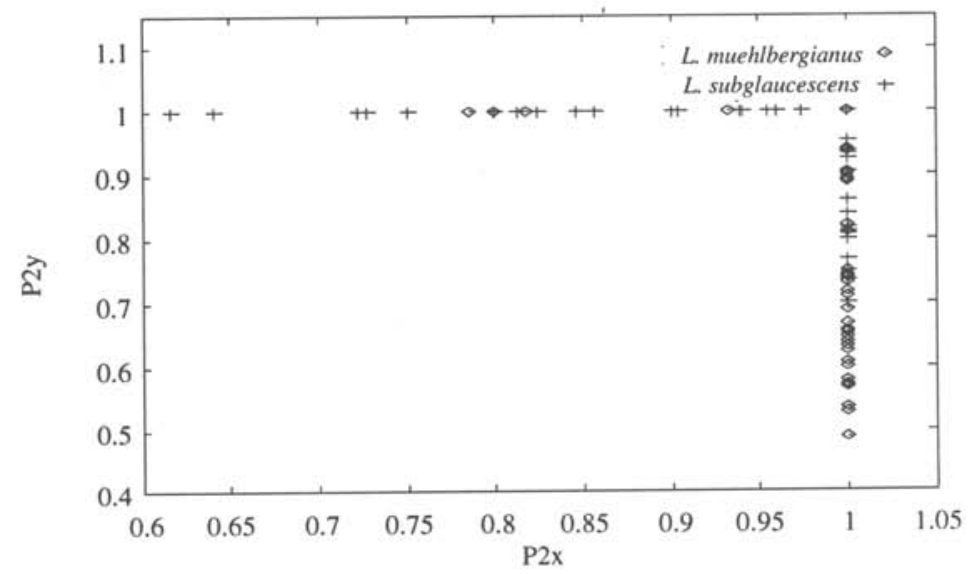

Figura 12. Componentes do vetor de geometria $\mathrm{P}_{2}$.

classes é feita no próprio espaço do descritor $\mathrm{R}_{1}$. Embora o erro na separação das espécies seja maior para o vetor $\mathrm{R}_{1}$ isoladamente (caso 4 ) do que o obtido pelo uso de todos os elementos da representação (caso 1), existem vantagens em sua aplicação, dado que para a separação não é necessária a redução dimensional, tornando mais intuitiva a compreensão do processo discriminatório.

$\mathrm{O}$ vetor $\mathrm{R}_{1}$, que neste caso pode ser considerado o elemento discriminador dominante, contém informações implícitas sobre o ângulo de inserção e o curso da nervura e, mesmo considerado isoladamente, caracteriza-se como um elemento de maior potencial discriminatório quando comparado com o ângulo de inserção, tradicionalmente utilizado na taxonomia. 
Tabela 1. Probabilidades de classificação errada de um foliolo da espécie A como sendo da espécie B.

\begin{tabular}{|c|c|c|c|}
\hline \multicolumn{4}{|c|}{ Probabilidade de classificação errada (\%) } \\
\hline & Espécie A & L.muehlbergianus & L.subglaucescens \\
\hline & Espécie B & L.subglaucescens & L.muehlbergianus \\
\hline Caso & Elementos & & \\
\hline 1 & $\mathrm{R}_{1}, \mathrm{R}_{2}, \mathrm{P}_{2}$ & $3,19 \%$ & $1,95 \%$ \\
\hline 2 & $R_{1}, R_{2}^{2}$ & $3,23 \%$ & $1,97 \%$ \\
\hline 3 & $\mathrm{R}_{1}, \mathrm{P}_{2}^{2}$ & $3,81 \%$ & $2,52 \%$ \\
\hline 4 & $\mathrm{R}_{1}{ }^{2}$ & $4,29 \%$ & $2,45 \%$ \\
\hline
\end{tabular}

Embora para alguns casos, como o das espécies aqui tratadas, possam existir discriminadores dominantes, a caracterização global da nervura e a discriminação é melhor realizada com base em todos os vetores de geometria. Esta afirmação pode ser verificada pela comparação dos resultados da Tab. 1, onde a proposição 1 apresenta o menor erro de classificação. A separação apenas com o uso do vetor $R_{1}$, portanto, não deve ser generalizada, embora válida para a identificação de Lonchocarpus muehlbergianus e L. subglaucescens.

Neste trabalho foi discutida a utilização da representação polinomial de Hermite na modelagem e descrição do curso de nervuras secundárias de folíolos. O potencial discriminatório dos elementos associados a sua representação foi testado sobre duas espécies do gênero Lonchocarpus, mostrando-se satisfatório.

No caso da aplicação considerada, os vetores se mostraram como bons separadores para as duas espécies de plantas, sendo $\mathrm{R}_{1}$ o elemento mais representativo no conjunto de parâmetros dos descritores. Desse modo, para Lonchocarpus muehlbergianus e $L$. subglaucescens, a quantificação do vetor $\mathrm{R}_{1}$ é uma grandeza de caracterização das espécies.

O estudo realizado mostrou que um problema de discriminação de duas classes de nervuras, bastante semelhantes, foi resolvido de maneira satisfatória. Diante da flexibilidade que pode ser obtida na modelagem e do modo como os parâmetros de controle da forma podem ser observados e analisados, é razoável concluir que a utilização da representação de Hermite é uma boa opção no processo de caracterização de nervuras, em adição às técnicas usualmente utilizadas em taxonomia vegetal, podendo, ainda, ser estendida para outras aplicações na área da biologia.

\section{Referências bibliográficas}

Bookstein, F. L.; Strauss, R. E.; Humphries, J. M.; Chernoff, B.; Elder, R. L. \& Smith, G. R. 1982. A comment upon the uses of Fourier methods in systematics. Systematic zoology 31(1): 85-92.

Box, G. E. P. \& Hunter, J. S. 1978. Statistics for experimenters: an introduction to design, data analysis, and model building. New York. John Wiley \& Sons.

Dickinson, T. A.; Parker, W. H. \& Strauss, R. E. 1987. Another aproach to leaf shape comparisons. Taxon 36(1): 1-20.

Ferson, S.; Rohlf, F. J. \& Koehn, R. K. 1985. Measuring shape variations of two-dimensional outlines. Systematic Zoology 34(1): 59-68.

Foley, J. D.; Van Dam, A.; Feiner, S. K. \& Hughes, J. F. 1990. Computer graphics: principles and pratice. 2. ed. New York. Addison-Wesley. 
Heijden, G. W. A. M. van der \& Berg, R. G. van den 1997. Quantitative assessment of corolla shape variation in Solanum sect. Petota by computer image analysis. Taxon 46(1): 49-64

Hickey, L. J. 1973. Classification of the architecture of Dicotyledonous leaves. American Journal of Botany 60(1): 17-33.

Hickey, L. J. 1979. A revised classification of the architecture of Dicotyledonous leaves. In: C.R. Metcalfe \& L. Chalk, (Ed.) Anatomy of the Dicotyledons. 2. ed., vol. 1, Oxford. Claredon.

Kores,P. J. ; Molvray, M. \& Darwin, S. P. 1993. Morphometric variation in three species of Cyrtostylis (Orchidaceae). Systematic Botany 18(2): 274-282.

Lin, C.-S. \& Hwang, C.-L. (ed.) 1987. New forms of shape invariants from eliptic Fourier descriptors. Pattern Recognition 20(5): 535-545.

Mclellan, T. 1993. The roles of heterochrony and heteroblasty in the diversification of leaf shapes in Begonia dregei (Begoniaceae). American Journal of Botany 80(7): 796-804.

Melville, R. 1969. The terminology of leaf architecture. Taxon 25(5): 549-561.

Melville, R. 1976. Leaf venation patterns and the origin of the Angiosperms. Nature 224: 121-125.

Niklas, K. J. 1994. Plant allometry: the scaling of form and process. Chicago. The University of Chicago.

Plass, M. \& Stone, M. 1983. Curve-fitting with piecewise parametric cubics. Computer Graphics 17(3): 229-239.

Press, W. H.; Teukolsky, S. A.; Vetterling, W. T. \& Flannery, B. 1992. Numerical recipens in C. New York. Cambridge.

Rizzini, C. T. 1977. Sistematização terminológica da folha, Rodriguésia 42: 103-125.

Rogers, D. F. \& Adams, J. A. 1990. Mathematical elements for computer graphics. 2. ed. Singapure. McGraw-Hill.

Roth, A.; Mosbrugger, V.; Belz, G. \& Neugebauer, H. J. 1995. Hydrodynamic modelling study of angiosperm leaf venation types. Botanica Acta 108: 121-126.

Schalkoff, R. 1992. Pattern recognition. New York. John Wiley \& Sons.

West, J. G. \& Noble, I. R. 1984. Analyses of digitised leaf images of the Dodonaea viscosa complex in Australia. Taxon 33(4): 595-613.

Zhou, Y.-H.; Ling, L.-B. \& Rohlf, F. J. 1985. Automatic description of the venation of mosquito wings from digitized images. Systematic Zoology 3(34): 346-358. 